University of Massachusetts Amherst

From the SelectedWorks of Julian Tyson

1996

Real-time internal standardization with an axiallyviewed inductively coupled plasma for optical emission spectrometry

Julian Tyson

Juan C. Ivaldi 


\title{
Real-time internal standardization with an axially-viewed inductively coupled plasma for optical emission spectrometry
}

\author{
Juan C. Ivaldi ${ }^{\mathrm{a}, *}$, Julian F. Tyson ${ }^{\mathrm{b}}$ \\ ${ }^{a}$ The Perkin-Elmer Corporation, 761 Main Avenue, Norwalk, CT 06859-0293, USA \\ ${ }^{b}$ Department of Chemistry, The University of Massachusetts, Box 34510, Lederle Graduate Research Towers, Amherst, MA 01003-4510, USA
}

Received 28 March 1996; accepted 9 May 1996

\begin{abstract}
An evaluation of precision improvements using real-time internal standardization with an axially-viewed inductively coupled plasma (ICP) is presented. New findings are presented with respect to the nature of the noise in the analytical signals from the axial ICP. It is observed that a high degree of correlation exists in the line signals from the axial ICP. Using the yttrium ion line at $371.030 \mathrm{~nm}$ as the internal standard, the analytical precision after the application of real-time internal standardization is maintained between 0.1 and $0.2 \%$ relative standard deviation (RSD) for ion lines. Precision improvement factors of 3 to 4 are obtained by comparison with the uncorrected results. With atomic lines, real-time internal standardization using the yttrium ion line is less effective, yielding precision values between 0.2 and $0.7 \%$ RSD. The precision improvement factors for atomic lines are between 1.5 and. 3. Thus, real-time internal standardization provides significant improvements in the RSDs of the line signals. The limits of these improvements are explored and an equation is presented which yields the fundamental shot noise limit for precision. Shot noise limited precision is demonstrated. However, this is not possible for all elements using a single internal standard signal. The effectiveness of real-time internal standardization is shown to be dependent on the nature of the specific spectral line. With the axially-viewed ICP, the dominant phenomenon preventing the full benefit of internal standardization from being obtained is the amplitude of the noise in the line signals and not the degree of correlation between analyte and internal standard signals. A trend is observed for atomic transitions in which lower excitation energy is correlated with higher relative noise amplitudes. This finding is in contrast with previously published work on the radially-viewed ICP. An explanation of this result is proposed which takes into account the influence of vaporizing sample droplets in the observation volume.
\end{abstract}

Keywords: ICP; ICP-OES; Internal standard; Noise; Optical emission spectrometry

\section{Introduction}

It is well known that internal standardization is an effective means for improving the precision of the atomic emission signals from the inductively coupled plasma (ICP) [1-9]. To obtain the best results, it is

\footnotetext{
* Corresponding author at: Cetac Technologies Inc., 5600 South 42nd Street, Omaha, NE 68107, USA.
}

useful to have instrumentation in which the analyte signal and internal standard signal can be measured simultaneously so that real-time internal standard correction can be performed [1-3]. This has been demonstrated with conventional, radial ICP viewing using a custom segmented-array charge coupled device detection system [9]. The increasing importance of axiallyviewed ICPs for routine analysis [10-18] presents the need to examine the applicability of real-time internal 
standardization for this plasma viewing arrangement. In this work, the noise in the emission signals from the axially-viewed ICP is examined. Real-time internal standardization is applied to line signals arising from the introduction of a sample which was prepared to simulate the digest of a botanical material. A means for assessing the expected precision after real-time internal standard correction is presented. The calculation of expected precision is based on an equation which accounts for all the sources of shot noise in the final corrected emission signal.

\section{Experimental}

\subsection{Experimental conditions}

The experimental conditions are summarized in Table 1. Experiments were carried out using a Perkin-Elmer Optima 3000-XL axial ICP instrument. The instrumentation has been described previously [18-20]. All solutions were prepared in aqueous media. For all experiments, the normal spectral resolution mode of the spectrometer with the $62 \mu \mathrm{m}$ slit was used. A special version of the Conespray nebulizer $[21,22]$ which has a sapphire gas orifice diameter of $178 \mu \mathrm{m}$ was used. The plasma operating conditions and nebulizer were chosen to minimize matrix effects with axial viewing without significantly sacrificing detection power [18]. These conditions involve the use of higher powers and lower nebulizer gas flow rates as recommended by Mermet [23] for robust operation of the ICP system. An integration time of $10 \mathrm{~s}$ was used for precision measurements.

Table 1

Experimental conditions

\begin{tabular}{ll}
\hline Instrument & Optima 3000-XL \\
\hline Viewing mode & Axial \\
RF power/W & 1450 \\
Nebulizer gas flow $^{\mathrm{a}}$ & 0.7 \\
Auxiliary gas flow $^{\mathrm{a}}$ & 0.5 \\
Plasma gas flow $^{\mathrm{a}}$ & 15 \\
Solution flow rate $^{\mathrm{b}}$ & 2 \\
Nebulizer type & Modified conespray $^{\mathrm{c}}$ \\
\hline
\end{tabular}

All gas flows are for Ar in $1 \mathrm{~min}^{-1}$.

${ }^{b}$ Units of $\mathrm{ml} \mathrm{min}^{-1}$.

${ }^{c}$ See text for details.
Table 2

Concentration of elements in the solution prepared for the precision study

\begin{tabular}{lc}
\hline Element & Concentration $/\left(\mu \mathrm{g} \mathrm{ml}^{-1}\right)$ \\
\hline $\mathrm{Al}$ & 0.5 \\
$\mathrm{Ba}$ & 0.1 \\
$\mathrm{Ca}$ & 200 \\
$\mathrm{Cu}$ & 0.1 \\
$\mathrm{Fe}$ & 0.5 \\
$\mathrm{~K}$ & 100 \\
$\mathrm{Mg}$ & 20 \\
$\mathrm{Mn}$ & 0.1 \\
$\mathrm{Na}$ & 1 \\
$\mathrm{P}$ & 5 \\
$\mathrm{~S}$ & 20 \\
$\mathrm{Sr}$ & 0.5 \\
\hline
\end{tabular}

For the internal standard work, a solution was prepared which approximated the elemental concentrations resulting from a complete digest of $0.5 \mathrm{~g}$ of Citrus Leaves (Standard Reference Material 1572 certificate, National Institute of Standards and Technology) diluted to $100 \mathrm{ml}$. The solution was prepared in $2 \% \mathrm{HNO}_{3}$ from commercially available single element standards (PEPure and SPEX) with starting concentrations of $1000 \mu \mathrm{g} \mathrm{ml}^{-1}$ and $10000 \mu \mathrm{g} \mathrm{ml}^{-1}$. The solution composition is given in Table 2. Yttrium was added as the internal standard element to a concentration of $0.1 \mu \mathrm{g} \mathrm{ml}^{-1}$ in the final solution. Table 3 lists the elements and wavelengths used in the internal standardization study. The lines chosen were those from elements that could be considered as major or minor constituents in the sample type

Table 3

Data for spectral lines used in the internal standardization study

\begin{tabular}{lllllr}
\hline Element & Atom/ion & $N(\mathrm{~nm})^{\mathrm{a}}$ & $\begin{array}{l}\text { Ionization } \\
\text { energy /eV }\end{array}$ & $\begin{array}{l}\text { Excitation } \\
\text { energy /eV }\end{array}$ & $\begin{array}{l}\text { Energy } \\
\text { sum/eV }\end{array}$ \\
\hline $\mathrm{Al}$ & I & 309.271 & & 4.02 & 4.02 \\
$\mathrm{Ba}$ & II & 233.527 & 5.21 & 6.01 & 11.22 \\
$\mathrm{Cu}$ & I & 324.754 & & 3.82 & 3.82 \\
$\mathrm{Fe}$ & II & 259.940 & 7.90 & 4.77 & 12.67 \\
$\mathrm{~K}$ & I & 404.721 & & 3.06 & 3.06 \\
$\mathrm{Mg}$ & II & 279.079 & 7.65 & 8.86 & 16.51 \\
$\mathrm{Mn}$ & II & 257.610 & 7.43 & 4.81 & 12.24 \\
$\mathrm{P}$ & I & 213.618 & & 7.21 & 7.21 \\
$\mathrm{~S}$ & I & 180.669 & & 6.86 & 6.86 \\
$\mathrm{Y}^{\mathrm{b}}$ & II & 371.030 & 6.53 & 3.52 & 10.05 \\
\hline
\end{tabular}

Air wavelengths.

${ }^{b}$ Internal standard element. 
simulated. Also provided in Table 3 are the ionization and excitation energies for each ransition. The energy sum is defined as the sum of excitation and ionization energies for ion lines. For atom lines, the energy sum equals the excitation energy. The energy sum is relevant in the discussion of observed rends in the results.

\subsection{Data processing}

Multicomponent spectral fitting (MSF) [24,25] data processing was applied in all experiments. The algorithm has the advantage that flicker noise in the background is removed as a noise component in the final signal $[18,25]$. Spectra to construct the data matrix were collected for each analyte element using individual single element standards. From the data matrix and the concentration coefficients, the values for the intensities, needed in the shot noise computations, are readily obtained.

For real-time internal standard correction, the analyte signal is divided by the internal standard signal. A critical condition is that the analyte and internal standard signals be collected at the same instant in time. The integration times were manually specified in the software to assure simultaneous data collection. In this work, the internal standard signal is from yttrium at the $371.030 \mathrm{~nm}$ line. The precision is calculated as the relative standard deviation (RSD) of 30 consecutive readings.

\subsection{Calculation of limiting precision}

It is known from previous studies $[1-9,18]$ that the RSD of a signal arises from contributions of various noise sources. It has been recognized that the best possible precision is that which arises only from the total shot noise in the signal. Thus, it is useful to calculate the limiting precision in order to estimate the lower boundary for improvement of precision.

In this work, an equation is derived which accounts for all expected sources of shot noise including the photon shot noise in the net analyte signal, the background, interfering element contributions, and the detector shot noise. The contribution of flicker noise is not taken into account since it is anticipated that application of real-time internal standardization will remove flicker noise. This equation is analogous to the
RSD of the background (RSDB) expression developed in the work of Boumans [26] and used in detection limit studies $[18,25,27]$. The derivation of the equation used in this study is shown below.

During an integration period, $\boldsymbol{n}$ photoelectrons are collected at a single pixel photodetector:

$\sigma_{n}^{2}=n$

The variance, represented in Eq. (1) as $\sigma_{n}{ }^{2}$, is that arising from photon shot noise [28]. However, it is desirable to represent both $n$ and $\sigma_{n}{ }^{2}$ in terms of readout counts as shown in Eq. (2) and Eq. (3) respectively:

$n=z \tau / \beta$

$\sigma_{n}^{2}=\sigma_{z}^{2} \tau^{2} / \beta^{2}$

where $z$ is the net signal intensity in counts $\mathrm{s}^{-1}, \beta$ is the conversion factor from counts to photoelectrons in counts per photoelectron (also referred to as the shot noise coefficient), $\sigma_{z}^{2}$ is the variance from photon shot noise in (counts $\left.\mathrm{s}^{-1}\right)^{2}$ and $\tau$ is the integration time in seconds. Eq. (4) is obtained by solving for $\sigma_{z}^{2}$ after substitution of Eqs. (2) and (3) into Eq. (1):

$\sigma_{z}^{2}=z \beta / \tau$

For an ICP signal, there are multiple sources of uncorrelated noise and each will contribute to the final signal noise. Since shot noise is uncorrelated, the different sources of variance add together [29] to produce the total variance from shot noise as shown in Eq. (5):

$\sigma_{t}^{2}=\sigma_{a}^{2}+\sigma_{b}^{2}+\sum_{i} \sigma_{i}^{2}+\sigma_{d}^{2}$

where the subscripts denote the sources of variance. In Eq. (5), the subscript $t$ denotes the total variance, $a$ is the analyte photon shot noise contribution, $b$ is the background photon shot noise contribution, $i$ is the photon shot noise contribution of an interfering species, and $d$ is the shot noise contribution from the dark current of the detector.

After substitution of Eq. (4) into Eq. (5), application of the square root operation, and rearrangement, the final expression for RSDA, the shot noise limited precision of the analyte signal, is defined in Eq. (6):

$\operatorname{RSDA}_{\text {shot }}=\sqrt{\frac{\beta}{z_{a} \tau}\left(1+\frac{z_{b}+\sum_{i} z_{i}+z_{d}}{z_{a}}\right)}$ 
The terms are defined as follows: RSDA $_{\text {shot }}$ is the RSD of the analyte signal arising from shot noise only (unitless), $z_{a}$ is the net analyte intensity at peak maximum (counts s ${ }^{-1}$ ), $z_{b}$ is the net background intensity at peak maximum (counts $\mathrm{s}^{-1}$ ), $z_{i}$ is the net intensity from interfering element $i$ at peak maximum (counts $\mathrm{s}^{-1}$ ), and $z_{d}$ is the magnitude of the dark current signal (counts $\mathrm{s}^{-1}$ ). The value of $\beta$ for the axial viewing instrument used in this work was determined by a detection limit study and was found to be $\beta=0.15$. The procedure for obtaining $\beta$ has been presented in prior work [18].

After division of the analyte signal by the internal standard signal, the resulting signal contains the shot noise of both signals. Thus, the equation describing the limiting RSD in the final corrected signal is given as Eq. (7):

$\operatorname{RSDA}_{\text {shot }}^{\prime}=\sqrt{\mathrm{RSDA}_{\text {shot }}{ }^{2}+\mathrm{RSDY}_{\text {shot }}{ }^{2}}$

in which RSDA $_{\text {shot }}^{\prime}$ is the shot noise limited RSD of the analyte after real-time internal standard correction, $\mathrm{RSDA}_{\text {shot }}$ (defined by Eq. (6)) is the shot noise limited RSD of the analyte signal before internal standardization, and RSDY shot $_{\text {is }}$ the shot noise limited RSD of the internal standard signal, in this case from the element yttrium.

The use of Eqs. (6) and (7) constitutes an improvement over an RSDA equation presented in earlier work [9]. The assumption associated with the previously published equation is that contributions from signal sources other than analyte emission are negligible. This assumption may be applied for studies using multielement standards free of interferences and with analyte element concentrations at relatively high levels. However, typical samples usually contain interferences and will have a wide range of analyte element concentrations. Therefore, Eqs. (6) and (7) better characterize the expected precision results when real-time internal standardization is applied since no assumptions are made about which signals will contribute.

\section{Results and discussion}

\subsection{Correlation of line signals}

In a study of the background signals from an axially-viewed ICP [18], it was found that a high degree of correlation existed in the noise signatures of background emission signals. In order to determine whether this characteristic also extended to the line signals from analyte elements, an experiment was performed to examine the correlation between the $\mathrm{Mn}$ signal at $257.610 \mathrm{~nm}$ and the $\mathrm{Mg}$ signal at 279.079 nm. In Fig. 1, it is observed that a very high degree of correlation is present in the line signals from the axial ICP. The RSD of either signal by itself is $0.25 \%$ whereas the RSD of the signal produced by dividing one signal by the other is $0.06 \%$. Therefore, real-time internal standardization is useful for improving the precision of measurements with this ICP viewing mode.

\subsection{Internal standardization results}

For the simulated Cirus Leaves digest, three values of the RSD were computed for each spectral line: (1) the RSD of the unmodified signal, (2) the RSD of the signals after division by the internal standard signal, and (3) the theoretical limiting RSD calculated using Eqs. (6) and (7). These values are shown graphically in Fig. 2. The elements are ranked from left to right according to increasing energy sum (see Section 2).

The precision for all elements was improved when real-time internal standardization was performed using the yttrium $371.030 \mathrm{~nm}$ ion line signal as the

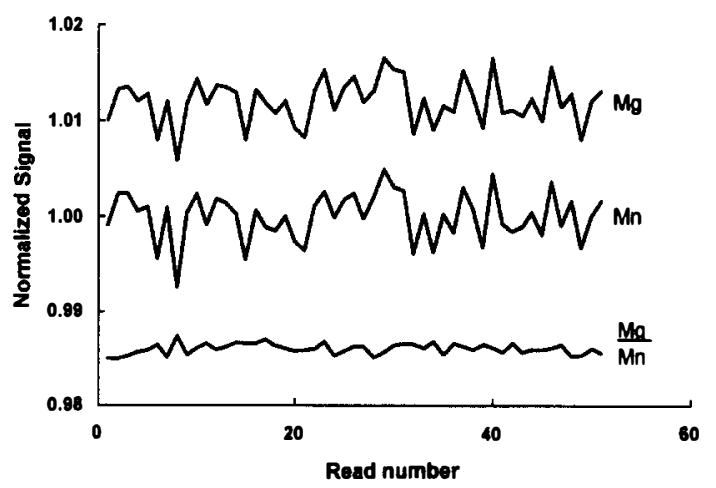

Fig. 1. A high degree of correlation is observed among the time resolved signals for two ion lines with the axially-viewed ICP. The $\mathrm{Mg}$ and Mn signals were collected simultaneously at the $\mathbf{2 8 0 . 2 7 0}$ $\mathrm{nm}$ and $257.610 \mathrm{~nm}$ lines respectively. The signals were normalized to 1 and offset from each other for clarity. The effectiveness of realtime internal standardization for precision improvement is observed by the reduction of correlated noise when the signal is presented as a ratio. The integration time per data point was $10 \mathrm{~s}$. 


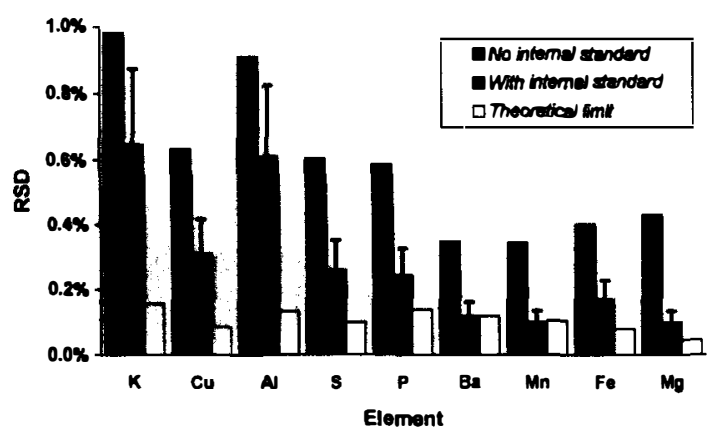

Fig. 2. Comparison of experimental and theoretical precision data based on 30 replicate measurements. The sample was a solution prepared to simulate digestion of a botanical material. Three RSD values are shown for each element, the unmodified RSD (No internal standard), the RSD after internal standardization with Y II $371.030 \mathrm{~nm}$ (With internal standard), and the theoretical shot noise limited RSD calculated from Eq. (6) and Eq. (7) (Theoretical limit). The 95\% confidence interval $(n=30)$ is shown for the precision values with internal standardization. The elements are arranged from left to right in order of increasing energy sum (see text for definition).

reference. For the ion lines, the RSD improves by factors of about 3 to 4 when real-time internal standard correction is applied. For the atomic lines, RSD improvement factors of about 1.5 to 3 are obtained.

For $\mathrm{Mn}$ and $\mathrm{Ba}, \mathrm{RSD}$ values after real-time internal standardization are indistinguishable from the calculated shot noise limited RSD. For $\mathrm{Fe}, \mathrm{Mg}, \mathrm{S}$, and $\mathrm{P}$, the RSDs after real-time internal standardization are between a factor of 1.5 and 2.5 of the shot noise limit. Finally, for $\mathrm{K}, \mathrm{Cu}$, and $\mathrm{Al}$, there is an RSD improvement after real-time internal standardization,

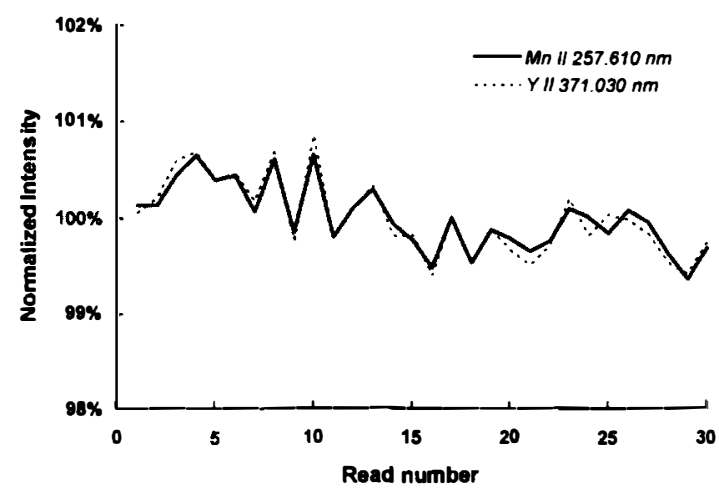

Fig. 3. Signal records for $\mathrm{Mn}$ and $\mathrm{Y}$ during the precision experiments. The two signals are highly correlated and have very similar noise amplitudes. Internal standardization gives the best results under these conditions. but the RSDs are as much as a factor 4 larger than the shot noise limit. None of the experimental values for the RSD are lower than the limiting RSD values calculated from Eq. (6) and Eq. (7). This result supports the postulate that the equations predict a fundamental lower limit for precision.

In order to determine if the differences in behavior for certain elements were caused by a lack of correlation between signals, correlation coefficients were calculated for each element using linear least-squares regression as described in prior work [3,9]. The correlation coefficients $\left(R^{2}\right.$ values) between yttrium and all other elements were found to be relatively high and ranged from 0.73 for $\mathrm{Al}$ to 0.95 for $\mathrm{Mn}$. Thus, the differences in the degree of correlation between the internal standard signal and the analyte signal do not convincingly explain the large range of results for the RSD after real-time internal standard correction.

It is observed in Fig. 2 that a trend related to energy sum exists. Lines with high energy sum have greater improvements in precision. Two extremes in Fig. 2 may be identified by comparing $\mathrm{Al}$, which gave poorer RSD results after real-time internal standardization, and $\mathrm{Mn}$, in which a shot noise limited RSD was achieved. To explore the differences further, the actual signal records were plotted for $\mathrm{Mn}$ in Fig. 3, and for $\mathrm{Al}$ in Fig. 4. Both $\mathrm{Mn}$ and $\mathrm{Al}$ give very similar noise signatures with respect to the yttrium signal. However, the important difference is that the Al signal has a greater noise amplitude by about a factor of 2 compared to yttrium. This difference in noise amplitude is largely responsible for the less than predicted

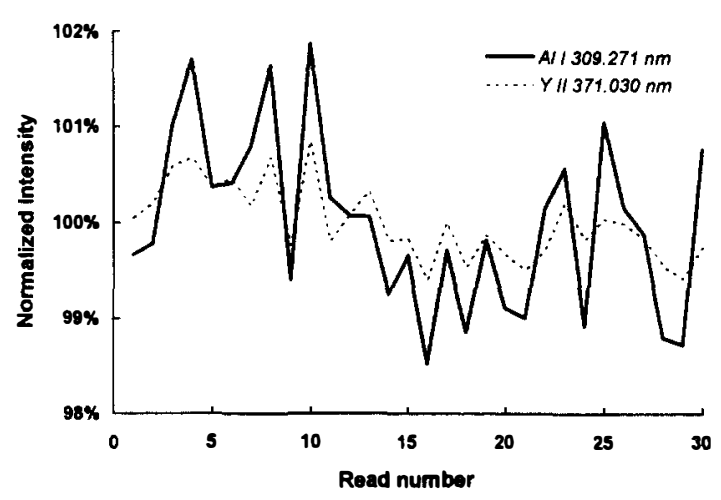

Fig. 4. Signal records for $\mathrm{Al}$ and $\mathrm{Y}$ during the precision experiments. The two signals are well correlated but have noise amplitudes which differ by a factor of about 2 . The difference in amplitude adversely affects the precision improvement with internal standardization. 
improvement of the RSD for $\mathrm{Al}$ after the internal standard signal division.

A value for the noise amplitude is easily obtained by computing the slope of the correlation plot $[3,9]$. Values other than unity for the slope result in a loss of effectiveness of real-time internal standardization and act as a source of error which prevents the achievement of shot noise limited precision. It is useful to refer to the slope of the correlation plot as the relative noise amplitude. The value of the relative noise amplitude for each element was computed and plotted in Fig. 5 as a function of the energy sum for the respective spectral lines. The energy sum is important since various trends with respect to this parameter have been previously reported in the ICP emission literature $[3,8,9,30]$.

Elements with lines having energy sums higher than that for the yttrium line $(>10 \mathrm{eV})$ generally had relative noise amplitudes near 1 . These same lines gave the best RSD results with real-time internal standardization, nearing shot noise limited performance. It is not surprising that this group of lines coincides with the ionic transitions, since these have the greatest energy sums. In contrast, elements with lines of lower energy sums than the yttrium line had relative noise amplitudes significantly greater than 1 . Poorer RSD results were obtained with this group of lines, which were all atomic transitions. For this group of lines (energy sums $<10 \mathrm{eV}$ ), a consistent trend was observed in which lower energy sum was well correlated with higher relative noise amplitude and

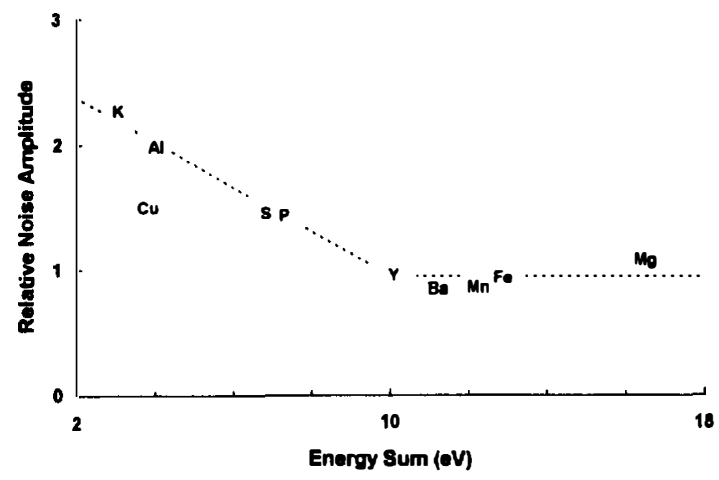

Fig. 5. A plot of relative noise amplitudes for the elements used in the axial ICP internal standardization study. Ionic lines all have similar noise amplitude and exhibit little dependence of the noise amplitude with energy sum. For atomic lines, the noise amplitude is found to be inversely proportional to the energy sum. lesser RSD improvements with real-time internal standardization. From Fig. 5, a nearly linear relationship is observed between energy sum and relative noise amplitude. The element $\mathrm{Cu}$ appears to be an unexplained outlier in the group.

The axial ICP results presented here for the atomic transitions contrast sharply with radial ICP data presented by Myers and Tracy [3]. First, the relative noise amplitudes for their experiments did not deviate more than about $10 \%$ from 1 regardless of the element or spectral line. In this work, values of the relative noise amplitudes exceeded 2 in the extreme case. Another contrast is that relative noise amplitudes of atomic lines examined in this study had a distinct trend with energy sum, whereas previously [3] no trend at all was observed. However, our results for ionic transitions indicate similar behavior to that reported by Myers and Tracy. It is very likely that the differences observed are the result of the different viewing modes (axial versus radial viewing) used in the two works. Differences in ICP conditions could also have been an influencing factor.

A possible explanation for the observed effects involves consideration of the fact that the sample is introduced to the ICP as a suspension of liquid aerosol droplets [31-34]. Fluctuations in the aerosol density give rise to the multiplicative flicker noise observed in the signals [1,3,31-33]. For example, when aerosol density increases, more analyte mass enters the ICP and the emission signals will increase in a proportional way. However, since the amount of solvent also increases, there is enhanced local cooling of the plasma in regions where a significant fraction of the sample is in the form of incompletely vaporized droplets. It is expected that such regions are imaged onto the spectrometer entrance slit because of the long pathlength of the axially-viewed ICP [18]. At the sub-millisecond time scale, atom emission is higher near droplets, whereas ion emission is higher when droplets are not present [31]. This is expected to preferentially increase atom emission signals at the moment when higher aerosol density passes through the observation volume. When the aerosol density increases, the local cooling effect near vaporizing droplets increases the fraction of analyte species that exist as atoms (as opposed to ions) [35]. This may explain why the noise amplitudes for atomic lines are larger than those observed for ionic lines when 
time-integrated measurements are made, as in this work. From these results, it is evident that the behavior of the noise in the emission signals from different spectral lines is an important consideration for the selection of the internal standard element line for axial ICP work.

Although not investigated here, it is possible to mathematically modify the yttrium reference signal so as to match the noise amplitude of the atomic transitions. Practical implementation of this approach hinges on the assumption that relative noise amplitudes are not strongly affected by plasma operating conditions. This will be examined in future work. Alternatively, an approach using multiple internal standards could be used $[4,5,7,8]$ in which more complex data processing is required. The later approach could include an atomic line as an internal standard to best match the requirements for analytes with atomic lines.

\section{Conclusions}

Line signals from the axial ICP are highly correlated as a function of time. For this reason, real-time internal standardization is very useful for improving the RSD of the line signals. Using the yttrium ion line at $371.030 \mathrm{~nm}$ as the internal standard, the analytical precision after the application of real-time internal standardization is maintained between 0.1 and $0.2 \%$ RSD for ion lines. Compared to the uncorrected results, precision improvement factors of about 3 to 4 are obtained. With atomic lines, real-time internal standardization using the yttrium ion line is less effective. Precision values between 0.2 and $0.7 \%$ RSD are obtained for the atomic lines after real-time internal standard correction. The precision improvement factors for atomic lines are between about 1.5 and 3 .

An equation for determining the lower limit to RSD improvement is presented. Achievement of shot noise limited precision is demonstrated. Real-time internal standardization improves the RSD for all element lines considered; however, the theoretical shot noise limit is not attainable for all of these. Clearly, the excitation characteristics of spectral lines are important to the quality of the RSD improvement obtained with real-time internal standardization. As reported by Myers and Tracy [3], a high degree of correlation is a necessary but not sufficient requirement. In this work with the axial ICP, the differences in excitation characteristics manifest themselves mainly as differences in noise amplitudes in the signal records. This is the primary source of variation in the RSD improvements for atom lines after real-time internal standard correction is applied.

Since the noise signatures are highly correlated, it is postulated that the same physical phenomenon is responsible for the observed fluctuations which appear as flicker noise in these signals. However, the magnitude of the response differs according to spectral line character. For atom lines, the noise amplitude is inversely related to the energy sum. For the ion lines, the noise amplitude is of similar magnitude with little dependence on the energy sum. The influence of incompletely vaporized droplets in the observation volume is believed to play an important role in the observed noise amplitude effects. In accordance with previous reports $[1,3,31-33]$, the main source of multiplicative flicker noise is believed to be the fluctuations in aerosol density originating in the sample introduction system.

\section{Acknowledgements}

We wish to thank Professor John Olesik of the Ohio State University for helpful discussions on the influence of sample introduction on ICP signals.

\section{References}

[1] R.M. Belchamber and G. Horlick, Spectrochim. Acta, Part B, 37 (1982) 1037.

[2] G.J. Schmidt and W. Slavin, Anal. Chem., 54 (1982) 2481.

[3] S.A. Myers and D.H. Tracy, Spectrochim. Acta, Part B, 38 (1983) 1227

[4] A. Lorber and Z. Goldbart, Anal. Chem., 56 (1984) 37.

[5] A. Lorber, Z. Goldbart and M. Eldan, Anal. Chem., 56 (1984) 43.

[6] G.F. Wallace, At. Spectrosc., 5 (1984) 5.

[7] M.H. Ramsey and M. Thompson, Analyst, 109 (1984) 1625.

[8] M.H. Ramsey and M. Thompson, Analyst, 110 (1985) 519.

[9] J.M. Mermet and J.C. Ivaldi, J. Anal. At. Spectrom., 8 (1993) 795.

[10] M.H. Abdallah, R. Diemiaszonek, J.J. Jarosz, J.M. Mermet, J. Robin and C. Trassy, Anal. Chim. Acta, 84 (1976) 271.

[11] F.E. Lichte and S.R. Koirtyohann, ICP Information Newslett., 2 (1976) 192. 
[12] D.R. Demers, Appl. Spectrosc., 33 (1979) 584.

[13] L.M. Faires, T.M. Bieniewski, C.T. Apel and T.M. Niemczyk, Appl. Spectrosc., 39 (1985) 5.

[14] J. Davies, J.R. Dean and R.D. Snook, Analyst, 110 (1985) 535.

[15] M.T.C. de Loos-Vollebregt, J.J. Tiggelman and L. de Galan, Spectrochim. Acta, Part B, 43 (1988) 773.

[16] M.T.C. de Loos-Vollebregt, J.J. Tiggelman, P.C. Bank and C. Degraeuwe, J. Anal. At. Spectrom., 4 (1989) 213.

[17] Y. Nakamura, K. Takahashi, O. Kujirai, H. Okochi and C.W. McLeod, J. Anal. At. Spectrom., 9 (1994) 751.

[18] J.C. Ivaldi and J.F. Tyson, Spectrochim. Acta, Part B, 50 (1995) 1207.

[19] T.W. Barnard, M.I. Crockett, J.C. Ivaldi and P.L. Lundberg, Anal. Chem., 65 (1993) 1225.

[20] T.W. Barnard, M.I. Crockett, J.C. Ivaldi, P.L. Lundberg, D.A. Yates, P.A. Levine and D.J. Sauer, Anal. Chem., 65 (1993) 1231.

[21] B.L. Sharp, J. Anal. At. Spectrom., 3 (1988) 613.

[22] J.C. Ivaldi, J. Vollmer and W. Slavin, Spectrochim. Acta, Part B, 46 (1991) 1063.

[23] J.M. Mermet, Anal. Chim. Acta, 250 (1991) 85.
[24] J.C. Ivaldi, D. Tracy, T.W. Barnard and W. Slavin, Spectrochim. Acta, Part B, 47 (1992) 1361.

[25] J.C. Ivaldi and T.W. Barnard, Spectrochim. Acta, Part B, 48 (1992) 1265.

[26] P.W.J.M. Boumans, Spectrochim. Acta, Part B, 46 (1993) 431.

[27] P.W.J.M. Boumans, J.C. Ivaldi and W. Slavin, Spectrochim. Acta, Part B, 46 (1991) 641.

[28] J.D. Ingle, Jr., and S.R. Crouch, Spectrochemical Analysis, Prentice Hall, New Jersey, 1988, pp. 141-142.

[29] J.D. Ingle, Jr., and S.R. Crouch, Spectrochemical Analysis, Prentice Hall, New Jersey, 1988, p. 146.

[30] M. Marichy, M. Mermet and J.M. Mermet, J. Anal. At. Spectrom., 2 (1987) 561.

[31] J.W. Olesik, L.J. Smith and E.J. Williamsen, Anal. Chem., 61 (1989) 2002.

[32] J.W.Olesik and S.J. Den, Spectrochim. Acta, Part B, 45 (1990) 731.

[33] J.C. Fister III and J.W. Olesik, Spectrochim. Acta, Part B, 46 (1991) 869.

[34] J.W. Olesik, J.A. Kinzer and B. Harkleroad, Anal. Chem., 66 (1994) 2022.

[35] J.W. Olesik, personal communication, 1995. 